\title{
INTERACTION OF COSMIC RAYS WITH A GALACTIC CORONA
} BOW-SHOCK

\author{
M.OSTROWSKI \\ Astronomical Observatory \\ Jagiellonian University \\ ul.Orla 171, 30-244 Kraków \\ Poland
}

\begin{abstract}
Basing on a simple 1-dim model, the process of diffusive particle acceleration at a galactic bow-shock is discussed.
\end{abstract}

Jokipii and Morfill $(1985,1987)$ suggested that a galactic wind terminal shock may be responsible for acceleration of cosmic rays (CR) up to ultra-high energies, $E \sim 10^{20} \mathrm{eV}$. A simple attempt to further study such mechanisms in the galactic halo is presented below for a galactic bow -shock which must be formed at the edge of galactic corona when it moves with a supersonic velocity in intergalactic medium. Thus, let us consider a (spiral) galaxy moving perpendicular to the galactic plane. The flow pattern of magnetized

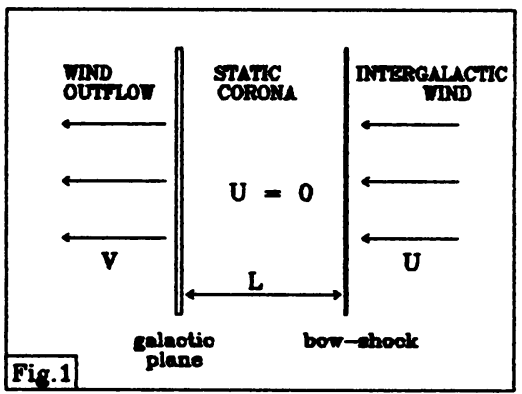
coronal and intergalactic plasma should be rather complicated, resembling partly the one observed in the Earth bow-shock. Here we consider a simplified situation presented in Fig.1. In this 1-dim model, we are able to describe a parallel to the shock advection of the plasma, carrying out imbedded energetic particles, in an approximate way: by introducing a particle loss term at the bow-shock. We will consider CR particles with much shorter mean free paths than the characteristic spatial scales of the system (a useful reference henceforth is a review by Drury (1983)). Assuming a constant diffusion

coefficient $D$ and constant plasma velocities $U$ and $V$, one can solve the stationary diffusion equation for the distribution function, $f(p, z)$. We assume that there is only one source of particles which is situated at the galactic plane: $q_{0}(p, z)=Q_{0} p^{-\alpha} H\left(p-p_{\min }\right) H\left(p_{\max }-\right.$ $p) \delta(z)$, where $H(x)$ is the Heaviside function; $Q_{0}, \alpha=$ const. It provides particles for further acceleration at the shock. Additionally, we assume a "monochromatic" particle loss term at the shock: $q_{L}(p, z)=-(3 D / U L) r f(p, z) \delta(z-L)$, where $r=$ const. With the use of jump conditions: $f=0$ and $D\left[\left(\frac{\partial f}{\partial z}\right)\right]+\frac{1}{3}[U] p\left(\frac{\partial f}{\partial p}\right)=-q(p)$ imposed at the velocity discontinuity at the galactic plane $\left(q=q_{0}\right)$ and at the shock $\left(q=q_{L}\right)$, and suitable boundary conditions $\int_{0}^{\infty} f(p, z) p^{2} d p<\infty$ and $f(p, z)>0$, one can solve the diffusion equation. Qualitative features of the solution are most clearly visible for a delta-like source, $\delta\left(p-p_{0}\right) \delta(z)$ (Fig.2). 

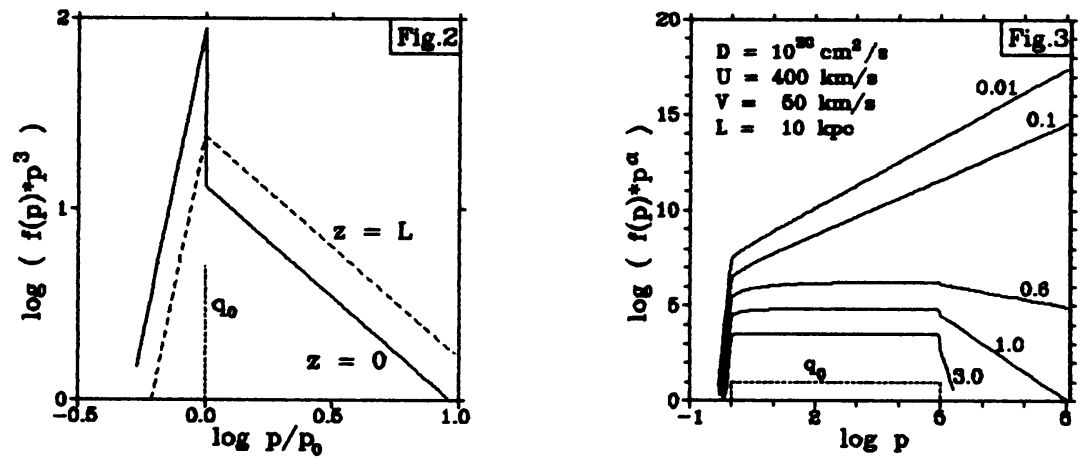

A set of solutions for the above power-law source $q_{0}(p)$ is presented in Fig.3 for few values of $r$; curves are shifted vertically by using different values of $Q_{0}$.A spectral index at highest energies, for $p>p_{\max }$, is $\lambda=0.5\left\{-(3-Y+X(1+r))-\left((3-Y+X(1+r))^{2}+12 Y+\right.\right.$ $\left.4 X Y r)^{1 / 2}\right\}(<-3)$ where $X \equiv 3 D /(U L)$ and $Y \equiv 3 D /(V L)$. It is an interesting fact that at the galactic plane particle anisotropy changes its sign within a momentum range $\left(p_{\min }, p_{\max }\right)$.

\section{Conclusions}

1. A galactic bow-shock may be responsible for extension of the cosmic ray energy spectrum above the claimed upper limit for the galactic disc: $E \sim 10^{15} \mathrm{eV}$ (Lagage and Cesarsky 1983). Flat, as well as steep spectrum can be formed at high energies. In our model, the high energy spectrum matches continuously to the spectrum in lower energies.

2. Particle dipole anisotropy arises in a natural way at the galactic plane. For isotropic diffusion its direction is determined by the relative position of the galaxy and a centre of the bow-shock.

3. The importance of the bow-shock acceleration should be considered seriously for galaxies in centres of rich galaxy clusters.

The author is grateful to Greg Morfill for suggesting the problem. The work was supported by Polish Interdisciplinary Project CPBP 01.03.

\section{References}

Drury, L.O'C., 1983, Rep.Prog.Phys. 46, 973.

Jokipii, J.R., Morfill, G., 1985, Astrophys.J. (Letters) 290, L1.

Jokipii, J.R., Morfill, G., 1987, Astrophys.J. 312, 170.

Lagage, P.O., Cesarsky, C., 1983, Astron. Astrophys. 125, 249. 